\title{
Propriedades e indicações clínicas das zircônias translúcidas: revisão narrativa
}

\author{
Clinical properties and indications of translucent zirconias: narrative review
}

Propiedades clínicas e indicaciones de circonias translúcidas: revisión narrativa

Nathalia Silveira Finck ${ }^{1}$, Mariana Itaborai Moreira Freitas ${ }^{2 *}$, Maria Eduarda Pereira de Oliveira ${ }^{1}$, Maria da Glória Bellotti², Victor Passamani Marques² ${ }^{2}$ Caio Felipe de Almeida Nobre ${ }^{2}$.

\section{RESUMO}

Objetivo: Realizar uma revisão narrativa atual sobre as principais propriedades e indicações do uso odontológico das zircônias translúcidas. Métodos: Foi realizada uma revisão de literatura com artigos disponíveis nas bases de dados PUBMED e MEDLINE, utilizando como termos de busca: cerâmicas odontológicas, zircônia, zircônia translúcida, zircônia estabilizada por ítrio. Resultados: Após a leitura dos artigos foi possível observar que a zircônia tetragonal estabilizada com ítria policristalina (Y-TZP) apresenta uma coloração branca opaca, trazendo deficiência em áreas estéticas, diante desta deficiência em mimetizar a estética do dente, diversas pesquisas aprimoraram as zircônias atualmente disponíveis na odontologia, a principal diferença encontrada foi no aumento da quantidade em mol de óxido de Ítrio, estabilizando essa zircônia na fase cubica (4Y-TZP e 5Y-TZP). Este aumento na adição do ítrio faz com que ocorra uma diminuição na tenacidade à fratura e resistência flexural, resultando em uma maior translucidez do material. Considerações Finais: As zircônias translúcidas são indicadas para procedimentos restauradores estéticos e contraindicadas para restaurações posteriores, devida a sua alta translucidez e baixa resistência mecânica. Contudo, mais estudos clínicos são necessários para determinar sua correta indicação.

Palavras-chave: Cerâmica, Propriedades físicas, Procedimentos clínicos.

\begin{abstract}
Objective: To carry out a current narrative review on the main properties and indications of the dental use of translucent zirconias. Methods: A literature review was performed with articles available in the PUBMED and MEDLINE databases, using as search terms: dental ceramics, zirconia, translucent zirconia, yttrium stabilized zirconia. Results: After reading the articles, it was possible to observe that the tetragonal zirconia stabilized with polycrystalline yttria (Y-TZP) presents an opaque white color, bringing deficiency in aesthetic areas. in dentistry, the main difference found was the increase in the quantity in mol of yttrium oxide, stabilizing this zirconia in the cubic phase (4Y-TZP and 5Y-TZP). This increase in the addition of yttrium causes a decrease in fracture toughness and flexural strength, resulting in a greater translucency of the material. Final Considerations: Translucent zirconias are indicated for aesthetic restorative procedures and contraindicated for posterior restorations, due to their high translucency and low mechanical resistance. However, further clinical studies are needed to determine its correct indication.
\end{abstract}

Keywords: Ceramics, Physical properties, Critical pathways.

${ }^{1}$ Rede Doctum, Serra - ES.

2 Universidade Estadual de Campinas (UNICAMP), Piracicaba - SP.

*E-mail: marianaitaborai@hotmail.com

SUBMETIDO EM: 7/2020

ACEITO EM: 8/2020

PUBLICADO EM: 11/2020 


\section{RESUMEN}

Objetivo: Realizar una revisión narrativa actual sobre las principales propiedades e indicaciones del uso dental de las circonias translúcidas. Metodos: Se realizó una revisión de la literatura con artículos disponibles en las bases de datos PUBMED y MEDLINE, utilizando como términos de búsqueda: cerámica dental, circonia, circonia translúcida, circonia estabilizada con itrio. Resultados: Después de leer los artículos, fue posible observar que la zirconia tetragonal estabilizada con itria policristalina (Y-TZP) tiene un color blanco opaco, lo que genera deficiencias en las áreas estéticas, debido a esta deficiencia en imitar la estética del diente, varios estudios han mejorado las zirconias disponibles actualmente En odontología, la principal diferencia encontrada fue el aumento en la cantidad en moles de óxido de itrio, estabilizando esta circonia en la fase cúbica (4Y-TZP y 5Y-TZP). Este aumento en la adición de itrio provoca una disminución en la resistencia a la fractura y la resistencia a la flexión, lo que resulta en una mayor translucidez del material. Consideraciones finales: Las circonias translúcidas están indicadas para procedimientos de restauración estética y están contraindicadas para restauraciones posteriores, debido a su alta translucidez y baja resistencia mecánica. Sin embargo, se necesitan más estudios clínicos para determinar su indicación correcta.

Palabras-clave: Cerámica, Propiedades físicas, Vías clínicas.

\section{INTRODUÇÃO}

Os materiais cerâmicos começaram a ser amplamente utilizados no final dos anos 60 , desenvolvidos especialmente para uso médico e odontológico. Essas cerâmicas foram denominadas biocerâmicas, devido sua boa biocompatibilidade com os tecidos moles, além de possuírem propriedades mecânicas satisfatórias. O desenvolvimento da zircônia como um biomaterial impulsionou a odontologia estética facilitando a reabilitação oral a base de cerâmicas puras. (VAGKOPOULOU T, et al., 2009). A zircônia foi introduzida na odontologia como um material CAD / CAM (Computer Aided Design/Computer Aided Manufacturing Desenho assistido por computador/Manufatura assistida por computador) em 1998 (BAN S, 2020).

O desenvolvimento de restaurações de cerâmicas puras, especialmente com a zircônia estabilizada com ítria (Y-TZP) como material de estrutura e os avanços das tecnologias CAD/CAM levaram ao aumento da utilização da zircônia estabilizada com ítria como material restaurador há cerca de duas décadas devido sua biocompatibilidade, excelentes propriedades mecânicas entre elas e a capacidade relevante de resistir a propagação de trincas. A odontologia também se tornou proficiente com diferentes aplicações baseadas em zircônia, por exemplo: próteses unitárias e parciais fixas, pinos endodônticos, implantes e braquetes ortodônticos (BAN S, 2020; ZHANG F, et al., 2015; ZHANG F, et al., 2020, VAGKOPOULOU T, et al., 2009).

A zircônia $\left(\mathrm{ZrO}_{2}\right)$ é quimicamente um óxido e tecnologicamente um material cerâmico, além de ser abundante na natureza (cerca de $0,02 \%$ da crosta terrestre) e insolúvel em água (LUGHI V e SERGO V, 2010). A zircônia $\left(\mathrm{ZrO}_{2}\right)$ é caracterizada como uma cerâmica policristalina, por apresentar menos de $1 \%$ de fase vítrea em sua composição, possui coloração esbranquiçada e apresenta uma característica denominada alotropia, isto é, a mesma composição química, porém seus grãos podem apresentar-se atomicamente distribuídos em formas cristalinas diferentes, sendo estas: monoclínica $(\mathrm{m})$, tetragonal (t) e cúbica (c) (KELLY JR e DENRY I, 2008).

A zircônia pura é estável em temperatura ambiente apresentando seus grãos estabilizados na fase monoclínica $(\mathrm{m})$. Este material mantém-se nesta fase $(\mathrm{m})$ em temperaturas menores que $1167^{\circ} \mathrm{C}$ e à medida que essa temperatura aumenta, seus grãos transformam-se em tetragonal ( $\mathrm{t}$ ) ou cúbica (c), sem variação da composição química do material. A forma monoclínica se converte para forma tetragonal a $1170{ }^{\circ} \mathrm{C}$, contraindo-se cerca de $4-5 \%$ do seu volume. A forma tetragonal se converte para a forma cúbica com cerca de $2370^{\circ} \mathrm{C}$ (CHEN YW, et al., 2016; MOQBEL NM, et al., 2019).

Cada fase proporciona à zircônia diferentes propriedades mecânicas e físicas. A fase tetragonal e a fase cúbica não são estáveis em temperatura ambiente, quando a temperatura diminui abaixo de $1167^{\circ} \mathrm{C}$, a zircônia retorna a sua fase monoclínica, não podendo ser utilizada clinicamente dessa forma. Por isso, há necessidade de adição de dopantes como por exemplo, o óxido de ítrio para a estabilização deste material nas fases tetragonal ou cúbica (CHEN YW, et al., 2016; MOQBEL NM, et al., 2019). 
A zircônia tetragonal policristalina estabilizada por ítria (Y-TZP) tradicionalmente utilizada na odontologia é a dopada com $3 \mathrm{~mol} \%$ de óxido de Ítrio (3Y) para estabilização da sua fase tetragonal ou cúbica em temperatura ambiente. Estes, são considerados materiais com alta resistência à flexão (900 - $1400 \mathrm{MPa}) \mathrm{e}$ alta tenacidade a fratura ( 5 a $10 \mathrm{Mpa} \mathrm{m}{ }^{1 / 2}$ ). Porém, a desvantagem deste material está na opacidade e dificuldade de mimetizar a translucidez do esmalte dentário, limitando seu uso na região anterior, onde a estética é altamente exigida, sendo normalmente utilizada como material para confecção de estruturas em próteses fixas em região posterior, implantes e pilares protéticos (ZHANG F, et al., 2015; ZHANG F ,et al., 2020).

Uma das opções para utilização da zircônia em regiões estéticas é a confecção de um copping de zircônia, sendo necessário utilizar cerâmica feldspática como cobertura, que possui alta translucidez e boas propriedades estéticas, contudo, são considerados materiais frágeis e com baixa resistência à fratura quando comparados com outros materiais cerâmicos. Por sua vez, esta característica mecânica deste tipo de revestimento não é suficiente para atuar como restaurador dentário, especialmente para os dentes posteriores (TUZZOLO NH, et al., 2018).

Foram observadas em estudos falhas clínicas como lascamento principalmente na porcelana, porém a base em zircônia era dificilmente fraturada, demonstrando que esse material desempenhava um núcleo resistente para peças protéticas (BA S, 2020; ZHANG F, et al., 2015). Outra explicação para as falhas entre restaurações é a presença de tensões residuais durante o processo de queima da camada de porcelana. Elas podem ocorrer devido a diferença de expansão térmica e/ou pela diferença de variação de temperatura durante o resfriamento da camada de porcelana (THOLEY MJ, et al., 2010).

A hipótese de utilização da zircônia monolítica surgiu como uma solução possível. Uma restauração monolítica 3Y-TZP tradicional seria muito opaca em comparação com os dentes naturais. Com intuito de aumentar a translucidez e a possibilidade clínica de utilizar restaurações monolíticas de zircônia, sem a necessidade de uma cerâmica feldspática de cobertura, foram desenvolvidas uma nova geração de zircônia. Foram adicionados uma maior quantidade de ítrio, $4 \%$ em mol (4Y-PSZ) e $5 \%$ em mol (5Y-PSZ) gerando zircônias parcialmente estabilizadas na fase cúbica (HATANAKA GR, et al., 2020; MOQBEL NM, et al., 2019).

No geral, para conseguir uma zircônia monolítica mais translúcida, uma das estratégias é utilizar a zircônia com grãos cúbicos, que são obtidas através do aumento do conteúdo de ítria. Em contrapartida, o aumento de translucidez e propriedades estéticas desses materias levou a redução nas propriedades mecânicas. Atualmente, as zircônia estão disponíveis em (3Y- TZP), (4Y- PSZ) e (5Y-PSZ) (ZHANG F, et al., 2015; MOQBEL NM, et al., 2019).

As zircônias (3Y- TZP), (4Y-PSZ) e (5Y-PSZ) apresentam graus de translucidez e valores resistência mecânica diferentes, portanto também possuem indicações clínicas diferentes. Sendo assim, o objetivo desse estudo foi descrever por meio de uma revisão narrativa, o desenvolvimento dos novos tipos de zircônias estabilizadas por ítrio nas últimas décadas, as propriedades mecânicas e características estéticas de cada tipo, assim como, determinar suas principais indicações para uso odontológico.

\section{REVISÃO BIBLIOGRÁFICA}

\section{Zircônia estabilizada por ítrio}

As demandas estéticas em ascensão para os dentes anteriores e posteriores estimulam o desenvolvimento de materiais restauradores, desde as tradicionais porcelanas fundidas ao metal até as restaurações totalmente em cerâmica. As cerâmicas vítreas tiveram seu uso limitado em apenas restaurações anteriores por conta de sua fragilidade. Porém, com avanço da tecnologia CAD / CAM e propriedades mecânicas, superiores às de outros sistemas cerâmicos, permitiu a zircônia estabilizada por ítria (Y-TZP) a sua utilização em restaurações extensas de cerâmica pura e em regiões posteriores. Essa indicação se dá principalmente, pelo efeito do endurecimento por transformação da zircônia tetragonal, conferindo dureza e resistência a esse material (ZHANG F, et al., 2015; RUALES-CARRERA, et al., 2019). 
O comportamento mecânico da zircônia tetragonal policristalina estabilizada por ítria (Y-TZP) pode ser modificado por transformações espontâneas dos grãos da fase tetragonal em fase monoclínica $(\mathrm{t} \rightarrow \mathrm{m})$ aumentando a tenacidade à fratura desse material. Quando se inicia uma trinca na superfície da Y-TZP, a concentração de tensão que ocorre na superfície gera uma transformação de fase do grão tetragonal em um grão monoclínico, associada com uma expansão volumétrica de 3 a 5\%. Essa transformação induzida por tensão, gera uma tensão compressiva ao redor dessa trinca em propagação, que é capaz de proteger a ponta da trinca, impedindo sua propagação. Esse mecanismo é conhecido como tenacificação por transformação de fase (LUGHI V e SERGO V, 2010).

Essa expansão, pode ocasionar uma diminuição de propagação de tricas neste material (aumento da tenacidade à fratura). Entretanto, estas transformações são desencadeadas por tensões externas como o bruxismo, ajustes nas peças com pontas muito grossas, carga mastigatória, abrasão por micro jateamento e envelhecimento hidrotérmico, denominada degradação hidrotérmica ou degradação a baixa temperatura (ZHANG F, et al., 2020). Condições específicas de processamento, umidade, estresse e temperaturas entre $150-400^{\circ} \mathrm{C}$, ocasionam espontaneamente um processo de degradação a baixa temperatura. A transformação espontânea e progressiva da fase tetragonal em monoclínica diminui suas propriedades mecânicas da Y-TZP em especial, sua resistência (ZHANG F, et al., 2020).

Essa degradação surge inicialmente em grãos superficiais, onde a água é incorporada aos grãos de zircônia preenchendo os espaços de oxigênio propagando-se para a superfície onde ocorre a transformação de fase $(\mathrm{m})$. O caráter triatômico do Ítrio favorece a presença de espaços vazios de oxigênio na estrutura da zircônia, facilitando a difusão das moléculas de água em sua massa (LUGHI V e SERGO V, 2010). O aumento de volume, que ocorre nessa transformação de tetragonal para monoclínico, provoca microfissuras, aumentando ainda mais a quantidade de água dentro dos grãos internos. Isso gera um aumento na rugosidade da superfície, levando a uma redução na resistência e na tenacidade a fratura (PEREIRA G, et al., 2015; LUGHI V e SERGO V, 2010).

Os grãos tetragonais da Y-TZP convencional são anisotrópicos e a refração da luz ocorre em diferentes direções cristalográficas, o que resulta em uma alta opacidade, limitando sua aplicabilidade clínica (HATANAKA GR, et al., 2018). Baseado nesta limitação foi desenvolvido estratégias para conseguir uma zircônia monolítica translúcida, uma delas é utilizar a zircônia com grãos cúbicos, que são isotrópicos e melhoram a transmitância da luz, estas são obtidos através do aumento do conteúdo de ítria associado a tratamentos térmicos específicos. No entanto, a zircônia cúbica apresenta uma redução no fenômeno de transformação de $t \rightarrow m$ (HATANAKA GR, et al., 2018; ZHANG F, et al., 2019).

A zircônia tetragonal diferente das cerâmicas cúbicas são opticamente anisotrópicas, implicando que o índice de refração é anisotrópico em diferentes direções cristalográficas, causando dispersão de luz, incluindo reflexão e refração nos limites dos grãos. A zircônia cúbica é isotrópica, possui cristais grandes, diminuindo a quantidade de vezes que a luz é dispersa, fazendo com que pareça mais translúcida (HATANAKA GR, et al., 2018; ZHANG F , et al., 2020; CAMPOSILVAN E , et al., 2018).

Com o aumento na adição do ítrio para a estabilização da zircônia na fase cúbica, há uma diminuição na tenacidade à fratura e resistência flexural, resultando em uma maior translucidez do material. Um fator que deve ser considerado quando elegemos a zircônia como material restaurador é a dureza deste material que, muitas vezes, está relacionada ao desgaste do dente antagonista à restauração e por isso, necessita de um adequado polimento do material (KWON S, et al., 2018; MAO L ,et al., 2018; PEREIRA GK, et al., 2018; AGREN M, et al., 2019; RUALES-CARRERA E, et al., 2019).

A translucidez da zircônia tem uma forte relação com sua microestrutura e composições químicas. Estudos anteriores mostraram que a translucidez da zircônia é influenciada pelo tamanho de grão da cerâmica Y-TZP, estruturas de contorno de grão e composição de fases. Os fabricantes eliminam ou reduzem a quantidade de alumina ou aumentam o teor de ítria para melhorar a translucidez da cerâmica Y-TZP. Entretanto, aumentar o conteúdo de ítria faz com que reduza as propriedades mecânicas da zircônia (AGREN M, et al., 2019; MOQBEL NM, et al., 2019; RUALES-CARRERA E, et al., 2019). O desafio atual está na fabricação de estruturas nanocristalinas de alta qualidade, com pouca ou nenhuma porosidade e defeitos. 


\section{Zircônia estabilizada com $3 \% \mathrm{~mol}$ de ítria - $3 Y$}

A tradicional e mais utilizada é a zircônia tetragonal estabilizada com $3 \%$ mol de ítria, sendo obtidas através de cristais finos de zircônia tetragonal com pequenas quantidades de estabilizadores de ítria (3Y-TZP) (MAO L, et al., 2018). As cerâmicas 3Y-TZP possuem alta resistência e tenacidade, porém apresentam propriedades ópticas desfavoraveis, como baixa translucidez. Suas principais indicações estão na confecção de restaurações de cerâmicas puras extensas em regiões posteriores (ZHANG F, et al., 2015).

No entanto, as cerâmicas 3Y-TZP são susceptíveis ao envelhecimento hidrotérmico o que resulta em maiores taxas de desgaste, rugosidade de superfície, degradação estética, perda de propriedades mecânicas e até mesmo falhas catastróficas, como o lascamento. A estabilidade ao longo prazo da cerâmica 3Y-TZP é crucial para manutenção de suas propriedades mecânicas (ZHANG F, et al., 2015).

Para melhorar as propriedades de resistência ao envelhecimento da 3Y-TZP, foi adicionado uma pequena quantidade de alumina. Porém, a presença da alumina gera uma desvantagem em relação as propiedades estéticas desse material, que é a opacidade (ZHANG F, et al., 2020). A adição da alumina no processo de sinterização da zircônia é necessária para reduzir a porosidade e eliminar aditivos de sinterização (ZHANG Y, et al., 2014).

A quantidade de alumina adicionada é geralmente de $0,25 \%$ em peso, mas quantidades mais baixas de alumina $(0,1$ ou $0,05 \%$ em peso) são usadas em novas gerações de cerâmicas de alta translucidez. Embora a redução da quantidade de adição de alumina possa aumentar a translucidez, a estabilidade hidrotérmica é simultaneamente reduzida (ZHANG F, et al., 2015; ZHANG F, et al., 2020). Além disso, a zircônia e a alumina têm diferentes índices de refração e o conteúdo de alumina pode diminuir a transmissão de luz em linha quando adicionada à zircônia (KWON S, et al., 2018). Como resultado, marcas comerciais desenvolveram zircôrnia com traslucidez cada vez maior, sendo elas: Lava Plus, Ivoclar Zenostar, Noritake Katana entre outras (ZHANG Y, et al., 2014) (Quadro 1).

Quadro 1 - Tabela representando o sistema cerâmico, marca comercial, indicações clínicas e resistência flexural (MPa), seguindo a ordem: zircônias estabilizadas por itria (3Y, 4Y e 5Y).

\begin{tabular}{|c|c|c|c|c|}
\hline $\begin{array}{l}\text { Zircônias } \\
\text { estabilizadas } \\
\text { por ítria }\end{array}$ & $\begin{array}{l}\text { Sistemas } \\
\text { cerâmicos }\end{array}$ & Marca Comercial & Indicação clínica & $\begin{array}{l}\text { Resistência } \\
\text { flexural } \\
\text { (MPa) }\end{array}$ \\
\hline $3 Y$ & $\begin{array}{l}\text { Segunda } \\
\text { Geração }\end{array}$ & $\begin{array}{c}\text { e.max }{ }^{\circledR} \text { Zircad LT e MO, } \text { Litana } \\
\text { KM } H T, \text { LAVA }{ }^{\mathrm{TM}} \text { Plus, } \\
\text { Bruxzir® }\end{array}$ & $\begin{array}{l}\text { Indicada para coroas } \\
\text { monolíticas e próteses } \\
\text { fixas em dentes } \\
\text { posteriores. }\end{array}$ & $1000 \pm 200$ \\
\hline $4 \mathrm{Y}$ & $\begin{array}{l}\text { Terceira } \\
\text { Geração }\end{array}$ & $\begin{array}{l}\text { Zpex }^{\circledR} 4, \text { Katana }^{\mathrm{TM}} \text { ST/STML, } \\
\text { IPS e.max } \\
\end{array}$ & $\begin{array}{l}\text { Indicada para coroas } \\
\text { monolíticas e próteses } \\
\text { fixas em dentes } \\
\text { anteriores. }\end{array}$ & $750 \pm 100$ \\
\hline $5 Y$ & $\begin{array}{l}\text { Terceira } \\
\text { Geração }\end{array}$ & $\begin{array}{c}\text { Bruxzir Anterior, LAVA } \\
\text { Esthetic, Katana }{ }^{\mathrm{TM}} \text { UT/UTML, } \\
\text { Prettau Anterior, Cercon }{ }^{\circledR} \mathrm{XT} \text {, } \\
\text { Zpex Smile }\end{array}$ & $\begin{array}{l}\text { Indicada para coroas } \\
\text { monolíticas e próteses } \\
\text { fixas em dentes } \\
\text { anteriores. }\end{array}$ & $600 \pm 50$ \\
\hline
\end{tabular}

Fonte: Finck NS, et al., 2020.

\section{Y-PSZ E 5Y-PSZ}

A zircônia estabilizada com cerca de 5\% mol de ítria (5Y) foi aplicada na odontologia em 2015 e contém uma quantidade maior de ítrio (5 mol\% tamanho de grão reduzido e maior quantidade de fase cúbica) (STAWARCZYK B, et al., 2017; PEREIRA G, et al., 2018). 
Este material não está apenas metaestável na fase tetragonal, porém também apresenta uma proporção de fase cúbica de até $53 \%$, consequentemente, uma pequena quantidade de transformação dos cristais ocorre, resultando em baixas propriedades mecânicas, especialmente com relação à resistência à propagação de trincas, sendo a resistência mecânica de $5 Y$ quase metade da $3 Y$ devido à redução da fase tetragonal da zircônia, ou seja, há uma menor tenacidade a fratura do material com escassos dados atuais sobre as propriedades mecânicas na literatura (KWON S, et al., 2018).

No entanto, mesmo os menores valores de resistência à fratura relatados são superiores às forças oclusais fisiológicas que podem ocorrer mesmo na região posterior, em torno de $900 \mathrm{~N}$. De qualquer forma, deve-se ter cautela ao usar zircônias com alto teor de óxido de ítrio em áreas ou situações com altas tensões. Os cristais cúbicos apresentam um volume maior em comparação aos tetragonais.

Isso significa que a luz se espalha menos fortemente nos limites dos grãos e nos poros residuais, tornando o material mais translúcido (STAWARCZYK B, et al., 2017; KWON S, et al., 2018; ELSAYED A, et al., 2019). Por isso, este material é utilizado principalmente, em restaurações anteriores, tendo em vista que são materiais com alta translucidez devido à maior fração da fase cúbica e menor fração da fase tetragonal da zircônia (ZHANG F, et al., 2020).

Em 2016, foi introduzido no mercado a zircônia estabilizada com cerca de 4\%mol de ítria (4Y) (BAN S, 2020). Um método para melhorar a translucidez da zircônia é introduzir a fase cúbica opticamente isotrópica na zircônia tetragonal (ZHANG F, et al., 2020). Isso foi alcançado utilizando um teor mais alto de ítria para produzir zircônias parcialmente estabilizadas, $4 \% \mathrm{em} \mathrm{mol} \mathrm{(4Y-PSZ).} \mathrm{No} \mathrm{entanto,} \mathrm{a} \mathrm{fase} \mathrm{cúbica} \mathrm{resulta} \mathrm{em}$ menor resistência e tenacidade, como já relatado anteriormente.

$\mathrm{Na} 4 \mathrm{Y}$ ainda há fase predominantemente mais tetragonal que a fase cúbica, portanto alguma resistência à tenacidade, dada pela transformação da zircônia, ocorre dependendo de onde a fissura começa na fase tetragonal ou cúbica. Isso explica por que há redução na resistência à fratura em $4 Y$ que sofreram envelhecimento artificial comparados àquelas de mesma composição que não passaram o processo de envelhecimento artificial (ELSAYED A, et al., 2019). Em 2017, foi introduzido no mercado a zircônia translúcida parcialmente estabilizada com cerca de 6\%mol de ítria (6Y) (BAN S, 2020).

\section{Adesão em zircônia}

A zircônia possui uma superfície apolar, quimicamente estável e inerte, o que dificulta estabelecer um protocolo de adesão à superfície deste material. A Y-TZP é uma cerâmica que não apresenta sílica e fase vítrea em sua composição, tornando-a resistente a ácidos que promovem o condicionamento superficial das cerâmicas antes da cimentação da peça protética.

Portanto, foram propostos métodos alternativos de condicionamento, sendo estes micromecânico e químico, destacando-se a abrasão de partículas de ar (MOQBEL NM, et al., 2019; RUALES-CARRERA E, et al., 2019).

Foram explorados métodos alternativos de condicionamento de superfície da zircônia, devido a essa dificuldade de gravar a superfície com ácidos. A retificação de superfície é uma alternativa comumente usada, podendo ser feita usando papel abrasivo ou rodas ( $\mathrm{SiC}$ ou $\mathrm{Al}_{2} \mathrm{O}_{3}$ ), abrasão por partículas de ar usando $\mathrm{Al}_{2} \mathrm{O}_{3}$ e lixar usando uma broca de diamante.

A vantagem desses métodos de retificação de superfície é que geralmente são fáceis de aplicar em um ambiente odontológico. No entanto, pesquisas demonstraram que as técnicas de retificação de superfície, usando cimentos de resina tradicionais, não têm efeito significativo no aumento da resistência de união da zircônia a cimentos de resina (MAROULAKOS G, et al., 2019).

A resistência da zircônia também pode ser influenciada por estes diferentes tratamentos de superfície, como a abrasão por micro jateamento com partículas de $\mathrm{Al}_{2} \mathrm{O}_{3}$ (que deve ser feito idealmente com partículas menores do que $50 \mu \mathrm{m}$ ) ou abrasão por micro jateamento com partículas de $\mathrm{Al}_{2} \mathrm{O}_{3}$ com revestimento de sílica (silicatização - partículas de óxido de alumínio revestidas por sílica) (MOQBEL NM, et al., 2019; RUALESCARRERA E, et al., 2019). 
Uma desvantagem com essas técnicas é que elas podem criar microfissuras de superfície. Essas falhas agem como locais de iniciação de trincas que podem diminuir a força e a resistência aparente à fratura (MAROULAKOS G, et al., 2019).

O jateamento provoca irregularidades na superfície e melhora a molhabilidade. Com isso, após este tratamento está indicado a utilização de um primer contendo monômeros fosfatados como o 10-MDP (10metacriloiloxidecildihidrogenofosfato). Isso porque o MDP favorecerá uma ligação iônica estável com a YTZP. Entretanto, caso o clínico esteja utilizando um sistema multicomponente, essa interação clínica entre a molécula MDP e o óxido de zircônio pode ser comprometida (LIMA RBW, et al., 2019).

Os cimentos que podem ser utilizados para cimentação de peças em zircônia são cimentos convencionais, sendo este o fosfato de zinco ou cimentos resinosos. Os cimentos resinosos promovem uma melhor integridade marginal da cimentação, além disso uma opção é combinar o primer com MDP com cimentos resinosos que também contenham MDP para aumentar a resistência de união da reabilitação oral (KERN M, 2015; LIMA RBW, et al., 2019).

\section{CONSIDERAÇÕES FINAIS}

As zircônias possuem diferentes indicações e limitações clínicas, por isso é necessário o conhecimento das diferentes apresentações da zircônia e suas marcas comerciais na clínica odontológica. As zircônias translúcidas são indicadas para procedimentos restauradores estéticos e contraindicadas para restaurações posteriores, devida a sua alta translucidez e baixa resistência mecânica. Contudo, mais estudos clínicos randomizados, prospectivos e controlados são necessários para determinar sua correta indicação.

\section{REFERÊNCIAS}

1. AGREN M, et al. Bond strength of surface-treated novel high translucent zirconia to enamel. Biomater Investig Dent, 2019; 6(1): 3542.

2. BAN S. Chemical durability of high translucent dental zirconia. Dent Mater J, 2020; 39(1): 12-23.

3. CAMPOSILVAN E, et al. Aging resistance, mechanical properties and translucency of different yttria-stabilized zirconia ceramics for monolithic dental crown applica-tions. Dental Materials, 2018; 34(6): 879-890.

4. CHEN YW, et al. Zirconia in biomedical applications. Expert Rev Med Devices, 2016; 13(10): 945-963.

5. ELSAYED A, et al. Influence of the yttrium content on the fracture strength of monolithic zirconia crowns after artificial aging. Quintessence Int, 2019; 50(5): 344-348.

6. HATANAKA GR, et al. The mechanical behavior of high-translucent monolithic zirconia after adjustment and finishing procedures and artificial aging. J Prosthet Dent, 2020; 123(2): 330-337.

7. KELLY JR, DENRY I. Stabilized zirconia as a structural ceramic: an overview. Dent Mater, 2008; 24(3): 289-98.

8. KERN, M. Bonding to oxide ceramics laboratory testing versus clinical out-come. Dental Materials, 2015; 31(1): 8-14.

9. KOLAKARNPRASERT N, et al. New multi-layered zirconias: Composition, microstructure and translucency. Dent Mater, 2019; 35(5): 797-806.

10. KWON SJ, et al. Comparison of the mechanical properties of translucent zirconia and lithium disilicate. J Prosthet Dent, 2018; 120(1): $132-137$.

11. LIMA RBW, et al. Effect of silane and MDP-based primers on physico-chemical properties of zirconia and its bond strength to resin cement. Dental Materials, 2019; 35(11): 1557-1567.

12. LUGHI V, SERGO V. Low temperature degradation-aging-of zirconia: A critical review of the relevant aspects in dentistry. Dental materials, 2010; 26(8): 807-820.

13. MAO L, et al. Graded ultra-trancent zirconia (5Y-PSZ) for strength and functionalities. J Dent Res, $2018 ; 97(11)$ : $1222-1228$.

14. MAROULAKOS G, et al. Effect of cement type on the clinical performance and complications of zirconia and lithium disilicate toothsupported crowns: A systematic review. Report of the Committee on Research in Fixed Prosthodontics of the American Academy of Fixed Prosthodontics. Prosthet Dent, 2019; 121(5): 754-765.

15. MOQBEL NM, et al. Influence of Aging on Biaxial Flexural Strength and Hardness of Translucent 3Y-TZP. Materials (Basel), 2019; 13(1): 27.

16. PEREIRA G, et al. Mechanical reliability, fatigue strength and survival analysis of new polycrystalline translucent zirconia ceramics for monolithic restorations. J Mech Behav Biomed Mater, 2018; 85: 57-65.

17. PEREIRA G, et al. Effect of low-temperature aging on the mechanical behavior of ground Y-TZP. J Mech Behav Biomed Mater, 2015; 45: 183-192.

18. RUALES-CARRERA E, et al. Adhesion behavior of conventional and high-translucent zirconia: Effect of surface conditioning methods and aging using an experimental methodology. J Esthet Restor Dent, 2019; 31(4): 388-397.

19. STAWARCZYK B., et al. (2017). Three generations of zirconia: From veneered to monolithic. Part I. Quintessence Int, 2017; 48(5): 369-380.

20. THOLEY MJ, et al. Thermal gradients and residual stresses in veneered Y-TZP frameworks. Dent Mater, 2011; 27(11): 1102-1110

21. TURON-VINAS M, ANGLADA M. Strength and fracture toughness of zirconia dental ceramics. Dent Mater, 2018; 34(3): 365-375.

22. TUZZOLO NETO H, et al. Laminated Veneers with Stratified Feldspathic Ceramics. Case Rep Dent, 2018; 6: 5368939.

23. VAGKOPOULOU T, et al. Zirconia in dentistry: Part 1. Discovering the nature of an upcoming bioceramic. Eur J Esthet Dent. 2009; 4(2): 130-151.

24. ZHANG F, et al. Highly-translucent, strong and aging-resistant 3Y-TZP ceramics for dental restoration by grain boundary segregation. Acta Biomater, 2015; 16: 215-222.

25. ZHANG F, et al. Importance of tetragonal phase in high-translucent partially stabilized zirconia for dental restorations. Dent Mater, 2020; 36(4): 491-500.

26. ZHANG Y. Making yttria-stabilized tetragonal zirconia translucent. Dent Mater, 2014; 30(10): 1195-1203. 